\title{
Meta-analysis genomics and interactomics data of relationship between the host and Bacillus anthracis
}

\author{
G.A. Pechkosvkii*, A.V. Abramovich \\ Stavropol Research Institute for Plague Control, Stavropol, Russia \\ *e-mail: grigorii.pechkovskii@gmail.com
}

Key words: host-pathogen interaction, Bacillus anthracis, data analysis

Motivation and Aim: B. anthracis passes in the host organism through the stages of development from spores into a vegetative cell. During this time there is a significant change in the genes expression of $B$. anthracis genes [1]. At present, a considerable amount of data has been collected. But there are still many mysteries about the life cycle of anthrax in the host. We have made an attempt to create pipeline for multiomics data analysis and applied it on genomic and interactomics $B$. anthracis data.

Methods and Algorithms: The pipeline was organized using custom python script. On the input of the pipeline, sequences are taken in the assemblies (from omics data) and the table to which groups this assembly belongs. The first stage in algorithm uses a BLAST for the selection of sequences with a certain degree of identity and places them in separate initial fasta files. Then groups them according to the specified cluster, makes a multiple alignment initial and grouped fasta files used MUSLE. The analysis used interactom data (IMEx IM-13779)[2], genomic data 36 complete RefSeq genome Bacillus cereus group, 12 genome well and 7 weakly susceptible to anthrax animal. Sequence was obtained from interaction data with the Uniprot id. And formed of a proteins assembly for $B$. anthracis and $H$. sapiens.

Results: Calculated the multiple alignment parameters (entropy, distance matrix), obtaining a set of intersecting clusters sequence. Common proteins in the $B$. cereus group 450 out of 936 with mean entropy 37,16 (SD 50,7), common proteins in B. anthracis cluster 854 out of 935 with mean entropy 4,25 (SD 25,5). Intersecting set proteins between well and weakly susceptible animal 925 out of 1638 with mean entropy 353,9 (SD 404) in well and 261,3 (SD 311,5) in weakly susceptible animal.

Conclusion: Thus, the obtained data suggest that quite a large number proteins $B$. cereus group is homologues to protein involved in the interaction between the host and $B$. antracis. But it has significantly more substitutions compared to the strain of B. antracis. Perhaps these proteins have undergone evolutionary changes to maintain pathogenicity. Also the values between well and weakly susceptible animal indicate a strong variety in protein. Probably, such data will be useful to understand inherited susceptibility to infection. Therefore, the evidence can help in the search for proteins that affect the course of anthrax disease. The data and script are available via email request.

\section{References}

1. Bergman N.H. et al. (2007). Transcriptional profiling of Bacillus anthracis during infection of host macrophages. Infection Immunity. 75(7):3434-3444.

2. Dyer M.D. et al. (2010). The human-bacterial pathogen protein interaction networks of Bacillus anthracis, Francisella tularensis, and Yersinia pestis. PloS One. 5(8):e12089. 